\title{
Orbital Lymphoma and Spontaneous Gastric Perforation, Rare and Fatal Complication
}

\section{Juan-Edric Gonzalez-Mondragon ${ }^{1}$, Luis Renato Vidaña Torres ${ }^{1}$, Adriana Dávila Camargo ${ }^{2 *}$}

${ }^{1}$ Ophthalmology Resident at Mexican Institute of Social Security, Torreon, Mexico

${ }^{2}$ Oculoplastics Department at Mexican Institute of Social Security, Torreon, Mexico

*Corresponding Author: Adriana Dávila Camargo, Oculoplastics Department at

Mexican Institute of Social Security, Torreon, Mexico.
Received: April 26, 2021

Published: May 22, 2021

(C) All rights are reserved by Adriana Dávila

Camargo.

\begin{abstract}
Orbital lymphomas represent $1 \%$ of non-Hodgkin lymphomas. Spontaneous bowel lymphoma perforation is rare and mostly associated with aggressive behavior. Diffuse large B-cell lymphoma is the most common lymphoma associated with intestinal perforation. We present a case report of a 56 years old patient who presented to our Oculoplastics department with a history of rapidly progressive proptosis and severe visual loss. After orbital lymphoma suspicion, a biopsy was conducted. During the post-operative period, the patient began with acute abdomen symptoms that required exploratory laparotomy. Multiple intestinal perforations were found through the surgery. Unfortunately, our patient evolved torpidly, presenting as well SARS-COVID 19 infection, perishing in the next days. In conclusion, even though a primary orbital lymphoma is suspected, we recommend deliberate systemic evaluation at the time of diagnosis and remembering COVID pandemic can worsen any hospitalized patient's prognosis.
\end{abstract}

Keywords: Orbit; Orbital Lymphoma; SARS CoV-2; Orbital Tumor; Gastric Perforation

\section{Introduction}

Lymphomas are a group of tumors derived from lymphoid tissue. They are classified into two major categories: Hodgkin Lymphoma (HL) and non-Hodgkin Lymphoma (NHL). The former arises from B-cell lymphocytes, whereas the latter has a more heterogeneous origin, emerging from $\mathrm{B}, \mathrm{T}$, and NK lymphocytes. Up to $85 \%$ of NHL arises from B-cell lymphocytes [1].

Ocular adnexal lymphomas (OAL) can emerge from the conjunctiva, eyelids, lacrimal gland, and orbital tissue, with the latter being the first one in frequency (50-60\%). The most common clinical characteristics associated with these tumors are soft tissue inflammation and irritation, pain, epiphora, proptosis and diplopia [2]. Diagnosis is made with a complete clinical history, physical examination, and imaging studies like computed tomography
(CT) and magnetic resonance (MRI) [3]. It's often necessary a multidisciplinary approach, where oncology, pathology, radiology, and ophthalmology specialists can be needed. Biopsy and immunohistochemistry studies make the definitive diagnosis. Some proposed treatments include surgical resection, radiotherapy, chemotherapy, steroids, and immunotherapy [1,3].

We present a case of a 56 years old male referred to our ophthalmology department with orbital lymphoma suspicion, which underwent spontaneous bowel perforation during the COVID-19 pandemic.

\section{Clinical Case}

A 56 years old male presents to our department with a red mass growing from the nasal conjunctiva on his right eye (OD). Previous- 
ly treated as pterygium, the patient noted a progressive and rapid mass growth, which limited eye movements, provoked proptosis, and diminished visual acuity.

The patient had a past medical history of smoking, suspended 12 years ago, and was diagnosed with diabetes mellitus 20 years ago, currently in treatment with insulin and oral hypoglycemics. The patient denied symptoms such as fever or night sweats. However, he did mention weight loss associated with a recent hospitalization two weeks prior, in another hospital facility, under the diagnosis of orbital cellulitis, treated with intravenous antibiotics, without improvement.

The ophthalmologic physical examination revealed a best-corrected visual acuity of the OD in no light perception and the left eye (OS) in 20/30. Intraocular pressure: OD $19 \mathrm{mmHg}$, OS $15 \mathrm{mmHg}$. The right eye presented with proptosis with axial displacement and exophthalmometry $24 / 16 \mathrm{~mm}$. Ocular adnexa presented with limitation to the palpebral closure as a result of chemosis and mild palpebral edema. Ocular movements of the right eye had a limitation of -4 in every direction, except abduction (Figure 1). Gonioscopy revealed open angles without vascular congestion at the Schlemm's channel. Anterior segment of the right eye presented with significant chemosis and proptosis, with a mydriatic pupil. The rest of the external exploration for the right eye remained within normal limits. OS did not have pathological signs. Funduscopy for the right eye revealed clear media, small optic nerve head, with "tenting" appearance, when comparing it to the contralateral eye. No optic nerve edema signs were present. Choroidal folds were evident at the posterior pole and macular area, in addition to intraretinal hemorrhages and soft exudates at the inferotemporal arcade (Figure 1). Funduscopy for the left eye revealed suggestive data for mild, non-proliferative diabetic retinopathy. Preauricular and submandibular ganglions were absent.

Previous orbital axial CT scan revealed a medial mass in the right orbit, with diffuse and irregular borders, that surrounded the globe without indenting it. Optic nerve straightening caused a tenting sign of the eye (Figure 2). Orbital apex exposed little space within it. After the evaluation, we suspected an orbital lymphoma and decided to undergo a biopsy.

On the day of the surgery, before the procedure, the patient presented with abdominal pain. A metabolic decompensation was
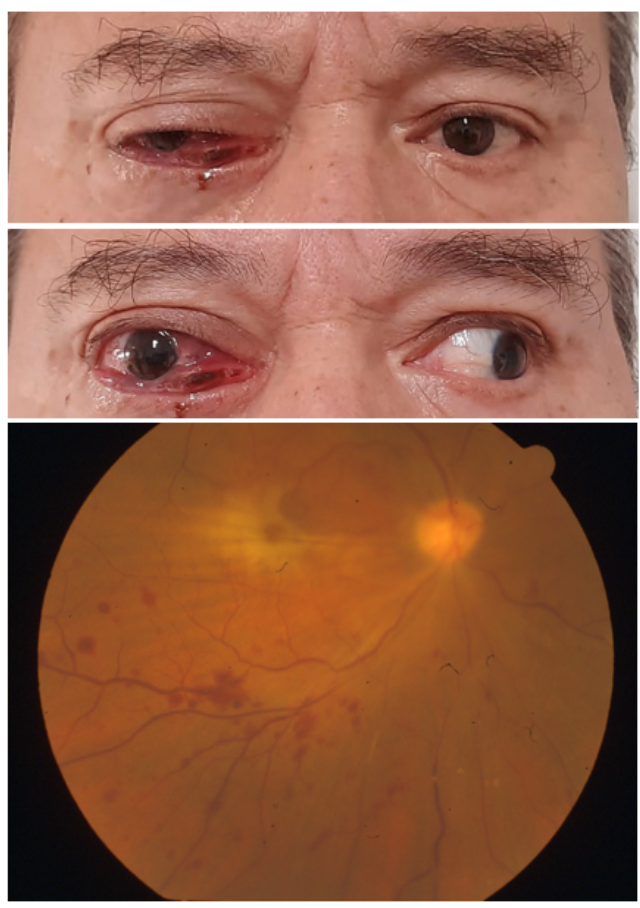

Figure 1: A) Primary position of the eyes B) OD with marked limitation to adduction C) Funduscopy of the OD, with a small optic disc, blurred borders, choroidal folds at the macular region, dot and flame-like hemorrhages at the inferior arcade, vein tortuosity, suggesting branch retinal vein occlusion.

found, with hyperglycemia up to $274 \mathrm{mg} / \mathrm{dl}$, blood pressure (BP) of $80 / 55 \mathrm{mmHg}$, and oxygen saturation $\left(\mathrm{SO}_{2}\right)$ of $93 \%$. The patient was stabilized and underwent incisional biopsy under general anesthesia. During the immediate post-op, the patient's abdominal pain persisted, adding abdominal distension.

We made an interconsultation to the general surgery department, which suggested an abdominal ultrasound. The imaging study revealed abdominal free fluid with the presence of punctiform echoes at the level of the right paracolic gutter.

The patient underwent urgent laparotomy, founding 1800cc of abdominal free fluid and multiple small bowel perforations. During the first post-op day, the patient persists with BP $80 / 55 \mathrm{mmHg}$, heart rate of $130 \mathrm{bpm}$, respiratory rate of $22 \mathrm{bpm}$ and a tempera- 


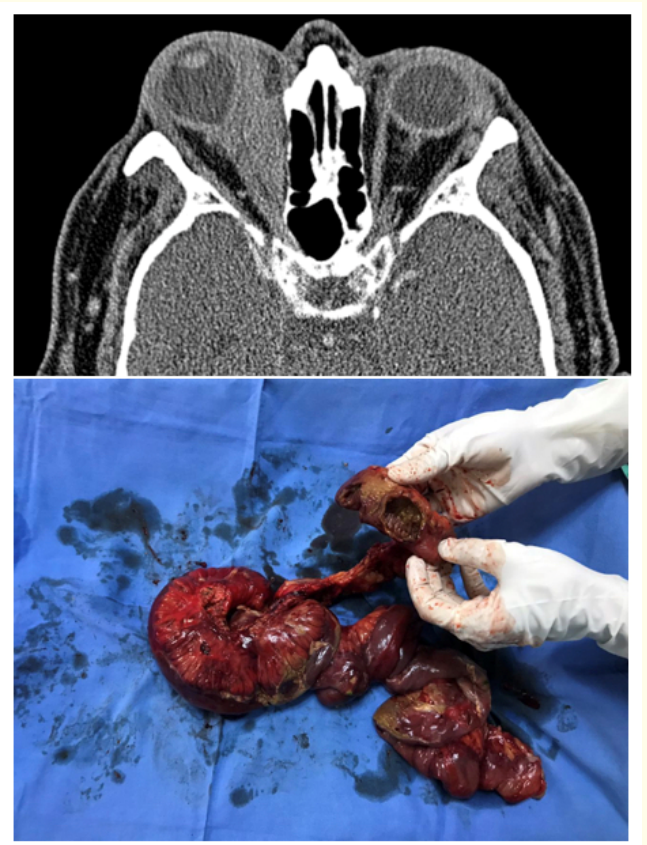

Figure 2: A) Axial view of an orbital CT scan, soft tissue window. Observe the medial mass with undefined borders that encircle the globe but don't compress it. Arrow: Tenting sign. B)

Jejunum and Ileum resection with multiple perforations.

ture of $36^{\circ} \mathrm{C}\left(96.8^{\circ} \mathrm{F}\right)$. We made an interconsultation to internal medicine, which diagnosed a circulatory shock and placed a central venous catheter with vasopressor therapy and antibiotic treatment based on vancomycin and meropenem. Laboratory findings revealed thrombocytopenia with $42 \times 10^{9} / \mathrm{uL}$ platelets (reference value: $150-400 \times 10^{9} / \mathrm{L}$ ). The patient received a fresh frozen plasma transfusion.

The patient progressed poorly and entered the intensive care unit with the diagnosis of mixed shock and multi-organic failure. The patient underwent intubation and control thorax radiography. The image revealed multiple areas of pulmonary infiltrates. Due to COVID-19 hospital protocol, we indicated a nasopharyngeal and oropharyngeal swab for sample collection and real-time polymerase chain reaction (rt-PCR) test for SARS-COV-2 detection.

Under the next hours, the patient developed severe hypoxia and mixed acidosis, needing advanced cardiorespiratory maneuvers.
Unfortunately, the patient perished in the following hours.

Rt-PCR confirmed COVID-19 infection. The pathology report of orbital, jejunum, and ileum confirmed diffuse B large cell, nonHodgkin lymphoma, with high malignancy grading.

\section{Discussion}

Orbital lymphomas account for only $1 \%$ of NHL. However, the orbit is the most commonly involved site for ocular adnexal lymphomas [2].

Several risk factors are associated with these tumors, being chromosome 3 and 18 trisomies one of the most common. Furthermore, chromosomal translocations as $\mathrm{t}(11 ; 18)(\mathrm{q} 21 ; \mathrm{q} 21)$ are associated with up to $40 \%$ of MALT (mucosa-associated lymphoid tissue) type ocular adnexal lymphomas. Some authors suggest $C$. psittaci infection as a causing agent [1,2]. Other risk factors as autoimmune diseases, immunosuppression, and smoking have also been mentioned [4]. Our patient had a precedent of 20 years of smoking.

The patient came to our department with signs, symptoms, and past medical history localized to the orbit. There is a higher incidence reported for primary orbital lymphomas over secondary lymphomas. Therefore, we suspected a primary orbital lymphoma and scheduled the patient for an incisional biopsy. We did not intentionally investigate for infirmities on another systemic level. Unfortunately, the clinical outcome was unfavorable for the patient.

Primary orbital lymphoma is that confirmed by biopsy, without suspicion or confirmed lymphoma in any other level. On the other hand, secondary lymphoma is that with simultaneous systemic disease or previous lymphoma diagnosis.

Up to $73 \%$ of B-cell orbital lymphomas have a primary origin, whereas $27 \%$ occur due to metastasis or adjacent invasion. The most common type is marginal zone extranodal lymphoma accounting for $60 \%$ of cases. Most of them, arising from a primary origin. Diffuse large B-cell lymphoma (DLBCL) is the second most common type, with up to $23 \%$ of cases, having $40 \%$ of these a secondary source [1]. The clinical case we present here had a secondary origin.

Several primary gastric lymphoma studies report spontaneous bowel perforation. It may present secondary to chemotherapy or 
necrosis of the sub-mucosa in non treated patients. DLBCL type is the most associated with intestinal perforation. Despite this, only $13 \%$ of the patients who develop these tumors present this complication. However, the presence of spontaneous bowel perforation is associated with higher mortality [5]. To the knowledge of the authors, there has not been a reported case as the one presented, in which lone orbital symptoms preceded intestinal perforations. Consequently, we might be describing the first known case of this kind.

The pathology department reported diffuse large B-cell lymphoma in both orbital and intestinal samples. Therefore, it's highly probable our patient's orbital tumor may be secondary to the intestinal affection.

Our patient had risk factors such as long-standing diabetes mellitus, smoking, and age. These made him vulnerable and at increased risk of severe illness from COVID-19 infection. Respiratory distress and the direct cause of death may be attributable to this infection, confirmed by rt-PCR [6].

Multiple treatments are available for these types of lymphomas, and the choice depends on the patient's clinical characteristics. Surgical resection is best for encapsulated tumors that can be removed at once. Nevertheless, they may reappear if no adjuvant therapy is indicated [2]. Radiotherapy is the treatment of choice for the majority of patients with ocular adnexal lymphomas. It can reach up to $85-100 \%$ of disease control. Several chemotherapy schemes are available, either alone or in combination with steroids and rituximab. However, the local recurrence rate and healing are worst if compared to radiotherapy $[1,2,8]$. In the presented case, given the patient's condition associated with bowel perforation, free abdominal fluid, and SARS COV-2 infection, starting radio or chemotherapy was not possible.

Some terminally ill patients with multiple comorbidities prevent invasive treatment. Although controversial for these cases, a "watch and wait" treatment scheme is preferred. In case of disease progression, invasive treatment begins [2]. Prognosis is generally good if treatment begins in the early stages, although the risk is particular for each patient.

For determining the prognosis of DLBCL, the revised International Prognostic Index has shown clinical utility. According to
Ann-Arbor classification, our patient catalogs as stage IV, A-E, because of its diffuse presentation, and B-symptoms and extranodal absence. At the same time, TNM classification indicates specific survival for OAL [10].

We decided to share this case because of the accelerated and complex clinical evolution. As discussed, our first suspicion was a primary orbital lymphoma because of the clinical data present at the time of diagnosis. The accelerated progression could have given us a hint of a more aggressive systemic disease, as well as the patient's symptomatology the day of the biopsy. Spontaneous bowel perforations are a rare complication in lymphoma patients. We have no knowledge of any other case reports in which orbital symptoms preceded intestinal perforations. SARS COV-2 infection may have limited even more our patient's prognosis. As ophthalmologists and orbital surgeons, we must remember how much orbital pathologies are associated with systemic diseases, and always be thorough in our examination. It is of utmost importance to remember the eyes are a window to multiorgan phenomena and can help us have a clearer view of the complete clinical picture.

\section{Conclusion}

In conclusion, even though a primary orbital lymphoma is suspected, we recommend deliberate systemic evaluation at the time of diagnosis and remembering COVID pandemic can worsen any hospitalized patient's prognosis.

\section{Declaration of Interest}

The authors have no conflict of interest to declare.

\section{Bibliography}

1. Olsen TG and Heegaard S. "Orbital lymphoma”. Survey of Ophthalmology 64 (2019): 45-66.

2. Stefanovic A and Lossos IS. "Extranodal marginal zone lymphoma of the ocular adnexa". Blood 114 (2009): 501-510.

3. Eckardt AM., et al. "Orbital lymphoma: Diagnostic approach and treatment outcome”. Journal of Surgical Oncology (2013): 11.

4. Munch-Petersen HD., et al. "Ocular adnexal diffuse large b-cell lymphoma: A multicenter international study". JAMA Ophthalmology 133.2 (2015): 165-173. 
5. Vaidya R., et al. "Bowel perforation in intestinal lymphoma: Incidence and clinical features". Annals of Oncology 24.9 (2013): 2439-2443.

6. Hanson KE., et al. "Last updated May 6, 2020 and posted online at www.idsociety.org/COVID19guidelines/dx. Please check website for most updated version of these guidelines". Supplementary materials are available here. Infectious Diseases Society of America G8uidelines on the Diagnosis of COVID-19 (2020).

7. Ahmed S., et al. "Orbital Lymphomas: A Clinicopathologic Study of a Rare Disease (2006).

8. Graue GF., et al. "Ocular Adnexal lymphoma staging and treatment: American joint committee on cancer versus Ann Arbor". European Journal of Ophthalmology: SAGE Journals 23.3 (2013): 344-355.

9. Sehn LH., et al. "The revised International Prognostic Index (R-IPI) is a better predictor of outcome than the standard IPI for patients with diffuse large B-cell lymphoma treated with RCHOP 109 (2007): 1857-1861.

10. Sung-Eun Lee., et al. "Feasibility of the TNM-based staging system of ocular adnexal extranodal marginal zone lymphoma of mucosa-associated lymphoid tissue (MALT lymphoma)". American Journal of Ophthalmology (2010).

Volume 4 Issue 6 June 2021

(C) All rights are reserved by Adriana Dávila Camargo. 